\title{
Restriction Fragment Length Polymorphism Mapping of Resistance to Two Races of Pyrenophora tritici-repentis in Adult and Seedling Wheat
}

\author{
R. J. Effertz, J. A. Anderson, and L. J. Francl
}

First and third authors: Department of Plant Pathology, North Dakota State University, Fargo 58105; and second author: Department of Agronomy and Plant Genetics, 411 Borlaug Hall, University of Minnesota, Minneapolis 55108. Accepted for publication 21 February 2001.

\begin{abstract}
Effertz, R. J., Anderson, J. A., and Francl, L. J. 2001. Restriction fragment length polymorphism mapping of resistance to two races of Pyrenophora tritici-repentis in adult and seedling wheat. Phytopathology 91:572-578.

Resistance to the chlorosis factor of tan spot of wheat, caused by the ascomycete Pyrenophora tritici-repentis, has been reported to be quantitative and a single quantitative trait loci (QTL), QTsc.ndsu-1A, explained $35 \%$ of the variation for resistance to a single isolate in seedlings of recombinant inbred (RI) lines derived from the cross W-7984/Opata 85. The objectives of this study were to determine the number and locations of genes conditioning resistance to the same isolate in adult plants of this

population and three isolates in seedlings of wheat RI lines derived from the cross W-7976/Trenton. An extensive restriction fragment length polymorphism map exists for the W-7984/Opata 85 population, and markers significantly associated $(P<0.01)$ with resistance to tan spot were selected to analyze the W-7976/Trenton population. A multiple regression model accounted for $49 \%$ of the variation for resistance in adult plants with $Q T s c . n d s u-1 A$, explaining $26 \%$ of the variation. QTsc.ndsu-1A explained 47,58 , and $64 \%$ of the variation for resistance in seedlings to isolates Pti2, 78-62, and D308, respectively. These results showed that the QTL for tan spot resistance on chromosome 1AS was effective in both seedlings and adult plants and against isolates from different races of $P$. tritici-repentis.
\end{abstract}

The fungus Pyrenophora tritici-repentis (Died.) Drechs., anamorph Drechslera tritici-repentis (Died.) Shoem., is the causal organism of the foliar disease tan spot of wheat (36). Tan spot is an economically important disease in the major wheat-producing areas of the world, including Australia and the Great Plains region of the United States and Canada $(13,31,44)$. A wide host range and the practice of conservation tillage, to conserve soil and soil moisture, contribute to the inoculum levels needed for serious epidemics $(13,15)$. Crop rotation, fungicide application, plowing or burning stubble, and genetic resistance are options available to control tan spot.

The symptoms caused by $P$. tritici-repentis on susceptible wheat cultivars consist of small dark brown infection sites with the development of necrotic lesions, with or without chlorotic halos, necrotic lesions with extensive chlorosis throughout the infected leaf, or extensive chlorosis without necrotic lesions $(16,17)$. Isolates of $P$. tritici-repentis have been grouped into five races based on their ability to induce tan necrosis, extensive chlorosis, both symptoms, or neither symptom on wheat differentials (21). Race 1 isolates can induce both tan necrosis and extensive chlorosis in susceptible wheat genotypes. Race 2 isolates can only induce necrosis, race 3 isolates can only induce extensive chlorosis, and race 4 isolates do not induce either symptom in susceptible genotypes and are considered avirulent. Race 5 isolates are similar in response to race 3 isolates, but are differentiated by the ability to induce chlorosis in cv. Katepwa and not in the line 6B365.

$P$. tritici-repentis produces several toxic compounds in culture that mimic the tan necrosis or extensive chlorosis symptoms in susceptible wheat lines $(1,3,12,30,39,41,42)$. The necrosis-inducing

Corresponding author: L. J. Francl; E-mail address: francl@badlands.nodak.edu

Publication no. P-2001-0330-01R

(c) 2001 The American Phytopathological Society proteinaceous toxin (Ptr ToxA) and its gene are well characterized. The amino acid composition and sequence have been determined, and the gene producing this protein has been cloned, sequenced, and its role in disease has been proven with classical and molecular genetics $(2,4,18,45)$.

The development of high levels of durable resistance in wheat cultivars that have acceptable agronomic qualities is the most desirable means to control tan spot. No commercially grown bread wheat cultivars possess complete resistance to tan spot and very few cultivars have high levels of resistance $(14,19,32,35)$. The inheritance of resistance to tan spot is both qualitative $(18,23,40)$ and quantitative $(7,25)$. Lamari and Bernier (18) studied segregation ratios of $F_{2}$ and $F_{3}$ populations and determined resistance to tan necrosis and extensive chlorosis were controlled by independent loci. They indicated that one recessive gene conditions resistance to tan necrosis and a single dominant gene conditions resistance to chlorosis with the possibility of modifiers acting in some populations.

Chromosomes $5 \mathrm{~B}$ and $1 \mathrm{~A}$ were indicated as contributing the most resistance to $P$. tritici-repentis when Rees and Platz (33) screened the Chinese Spring chromosome substitution series Kenya Farmer, Timstein, and Thatcher. Restriction fragment length polymorphism (RFLP) markers were detected by Faris et al. (8) for the Ptr ToxA insensitivity locus at 5.7 and 16.5 centimorgans (cM). Aneuploid analysis indicated that this locus resides on the long arm of chromosome 5B, and the symbol tsnl was given to designate this gene (8). The location of $t s n 1$ to chromosome 5BL was confirmed independently with Chinese Spring (Kenya Farmer) substitution lines and Chinese Spring aneuploids (38).

Faris et al. (9) used a recombinant inbred (RI) population derived from the cross of the resistant synthetic hexaploid W-7984 with the susceptible cv. Opata 85 to study the genetics of chlorosis induction. An extensive RFLP map exists for this population, which allowed for the detection of quantitative trait loci (QTL) 
(24,27-29,43). Lesion-type means regressed on RFLP data identified loci associated with resistance to chlorosis induction. Analysis indicated resistance was quantitative, with a major effect locus on chromosome $1 \mathrm{AS}$, a minor effect locus on chromosome 4AL, and an interaction between the 1AS locus and a locus on chromosome 2DL. The gene on 1AS explained $35 \%$ of the variation for resistance to chlorosis induction by $P$. tritici-repentis. A multiple regression model of the $1 \mathrm{AS}$ locus, $4 \mathrm{AL}$ locus, and the significant interaction explained $49 \%$ of the variation for resistance in seedlings of this population. The symbol QTsc.ndsu-1A was proposed to designate the major effect locus on 1AS (9).

Reports of the correlation of resistance to $\tan$ spot between seedlings and adults have not been consistent. Hosford et al. (14) stated that resistance is statistically similar between seedling and adult wheat. Riede et al. (35) reported that adult disease reactions do not always agree with wheat seedling evaluations for tan spot, and in Triticum turgidum var. durum, Fernandez et al. (10) reported that resistance to tan spot at the adult stage was not expressed at the seedling stage in some lines. This phenomenon also has been seen in the net blotch system between barley (Hordeum vulgare L.) and the fungal pathogen $P$. teres $\mathrm{f}$. sp. teres, in which Steffenson et al. (37) reported three QTLs associated with resistance at the seedling stage and seven QTLs associated with resistance at the adult stage. Cox and Hosford (5) determined that tan spot reactions on flag leaves of greenhouse-grown winter wheat closely reflected those seen on adult plants in the field.

Faris et al. (9) inoculated a subset (six resistant, six moderately resistant to moderately susceptible, and six susceptible) of the W7984/Opata 85 RI population with race 3 isolate D308 and found a high correlation $(r=0.81 ; P<0.01)$ with the Pti2 inoculations. However, resistance to the extensive chlorosis symptom has only been analyzed in detail for one RI population and one isolate of $P$. tritici-repentis. The objectives of this study were to determine if the QTsc.ndsu-1A locus that conditioned resistance in seedlings to chlorosis induction by $P$. tritici-repentis also conditioned resistance in adults of the same population and to determine whether $Q T s c . n d s u-1 A$ was associated with resistance to three isolates of $P$. tritici-repentis in a different RI population of wheat.

\section{MATERIALS AND METHODS}

Adult plant material. The wheat plants used in this experiment were from an RI population consisting of $135 \mathrm{~F}_{7}$-derived singleseed-descent lines from the cross of W-7984 (a synthetic hexaploid wheat derived from the cross of the durum parent cv. Altar 84 and Aegilops tauschii [Coss.] Schmal.) and cv. Opata 85, a hard red spring wheat from the International Maize and Wheat Improvement Center (CIMMYT, Mexico). This population was provided by M. E. Sorrells, Cornell University, Ithaca, NY. The synthetic hexaploid parent of this population showed high levels of resistance to tan spot in greenhouse studies (35). This population segregates for extensive chlorosis and is resistant to tan necrosis (9). Checks in the experiment included both parents, resistant cv. Erik, and susceptible lines ND495 (nec+, chl-) and 6B365 (nec-, chl+).

Seedling plant material. The wheat plants used were from an RI population consisting of $113 \mathrm{~F}_{5}$-derived single-seed-descent lines from the cross and reciprocal cross of W-7976 $\times$ Trenton. W-7976 is a synthetic hexaploid wheat with the pedigree Cando/R143//mexi 'S'/A. tauschii (C122) and was provided by A. Mujeeb-Kazi, CIMMYT. W-7976 was determined to be highly resistant in both the seedling and adult stages to tan spot (35). Trenton is a tan spot-susceptible hard red spring wheat cultivar developed at North Dakota State University and was provided by R. Frohberg. Cultivar Trenton exhibited symptoms of tan necrosis and extensive chlorosis to $P$. tritici-repentis. Checks included both parents, resistant cv. Erik, susceptible cv. Kulm (nec+, chl+), and susceptible lines ND495 (nec+, chl-) and 6B365 (nec-, chl+).
Inoculum preparation. The $P$. tritici-repentis isolates used in this study were race 1 isolate Pti2 (ATCC 44143), race 1 isolate 78-62 (ATCC 44142), and race 3 isolate D308 $(6,20)$. Inoculum was prepared from a single source of dried mycelial plugs of each isolate stored at $-20^{\circ} \mathrm{C}$. Inoculum was produced according to the method in Faris et al. (9). The conidia were used directly, or could be stored at $-20^{\circ} \mathrm{C}$, up to 1 month later with no noticeable decrease in infection rate.

Conidial inoculation and rating. For adult plants, two experiments with three replications of each RI line, parents, and checks were conducted in the greenhouse at an average temperature of $22^{\circ} \mathrm{C}$ with a photoperiod of $16 \mathrm{~h}$ and a light intensity of $900 \mu \mathrm{mol}$ photons $\mathrm{m}^{-2} \mathrm{~s}^{-1}$. To synchronize flowering, genotypes were planted at one of three planting dates, each 7 days apart, according to previously collected heading dates. This synchronization facilitated a single inoculation of the flag leaf on the entire RI population. In the first experiment, plants were grown in soil beds with $100 \mathrm{~cm}$ spacing between plants and $300 \mathrm{~cm}$ spacing between rows. In the second experiment, plants were grown in tree pots $(10 \times 10 \times 20 \mathrm{~cm})$ with a potting mix consisting of peat moss $(75 \%)$ and perlite $(25 \%)$. Ten seeds of each RI line were pregerminated in petri dishes and three of these were planted in the greenhouse beds or pots.

When 85 to $90 \%$ of the plants were at anthesis, they were inoculated with 3,000 conidia per ml. Plants were sprayed to run off with the conidial solution with a pressurized atomizer. The conidia solution was kept on ice and continually stirred to minimize germination before being sprayed on the plants. After inoculation, the plants were allowed to dry for $30 \mathrm{~min}$. A plastic tent was erected over the plants, and plants were misted for $24 \mathrm{~h}$ by several ultrasonic humidifiers. The average temperature and relative humidity in the tent over the 24-h period were recorded at $20.5^{\circ} \mathrm{C}$ and $100 \%$, respectively, with data logger sensors (CR10; Campbell Scientific, Logan, UT) placed in the wheat canopy.

For the W-7976/Trenton population, seedlings of each RI line, parents, and checks were grown in plastic cones containing a potting mix consisting of peat moss $(75 \%)$ and perlite $(25 \%)$ in the greenhouse at an average temperature of $22^{\circ} \mathrm{C}$ with a photoperiod of $16 \mathrm{~h}$ and a light intensity of $900 \mu \mathrm{mol}$ photons $\mathrm{m}^{-2} \mathrm{~s}^{-1}$ at plant height. Seven seeds of each RI line, parents, and checks were planted, and three plants from each entry were selected for the experiment based on similarity of growth stage. This was done to minimize age effects of inoculated leaves because younger leaves tend to be more resistant than older leaves (5). Each $P$. tritici-repentis isolate was used as inoculum in each of two experiments with three replications of each RI line, parents, and checks in a completely randomized design.

When the third leaf was fully emerged, plants were sprayed to run off with the conidial solution $(3,000$ conidia per $\mathrm{ml}$ ) as previously described. After inoculation, the seedlings were allowed to dry for $30 \mathrm{~min}$ and placed in a mist chamber at $20^{\circ} \mathrm{C}$ in the dark for $24 \mathrm{~h}$. The chamber was misted by an ultrasonic humidifier. After the 24-h wet period, the plants were placed in a growth chamber at $20^{\circ} \mathrm{C}$ and subirrigated to avoid further leaf wetting.

Seven days after inoculation, the flag leaf (for adults) or the third leaf (for seedlings) was rated for disease according to lesion type from 1 to 5 ( $1=$ most resistant and $5=$ most susceptible $)$ based on the scale developed by Lamari and Bernier (16). Analysis of variance was conducted to identify significant variation among RI lines, and variances were tested for homogeneity between blocks and experiments. RI lines that showed both highly resistant and highly susceptible reactions were eliminated from the analysis based on the possibility that they may be heterogeneous.

RFLP analysis of adult plants. An extensive RFLP map exists for the W-7984/Opata 85 RI population (24,27-29,43). A subset of 541 markers was selected to give the most complete genome coverage possible and used to identify associations with lesion- 
type scores. The computer program QGENE (version 2.25b) (26) was used to execute interval regression mapping (11) to infer the best position for QTLs between marker loci. Because missing data points existed, the "complete missing data" feature was used to estimate QTL from the flanking markers of the nearest nonmissing data points. All the loci with significant main effects were tested against all the other markers on the map to detect significant $(P<0.01)$ interactions. Markers showing significant main effects and interactions were used to identify the best multiple regression model, and markers that failed to retain significance in the full model were eliminated. The coefficient of determination $\left(R^{2}\right)$ is the proportion of the total phenotypic variance explained

TABLE 1. Lesion-type means of parents and checks after inoculation of flag leaves (adults) or third leaves (seedlings) with conidia from isolates of Pyrenophora tritici-repentis

\begin{tabular}{lccccc}
\hline & \multicolumn{4}{c}{ Isolate $^{\mathrm{z}}$} \\
\cline { 2 - 5 } Genotype & Adult & & \multicolumn{3}{c}{ Seedling } \\
\cline { 2 - 5 } \cline { 5 - 6 } & Pti2 & & Pti2 & D308 & $78-62$ \\
\hline W-7984 (resistant parent) & $1.3 \mathrm{a}$ & $\ldots$ & $\ldots$ & $\ldots$ \\
Opata 85 (susceptible parent) & $3.7 \mathrm{~b}$ & $\ldots$ & $\ldots$ & $\ldots$ \\
W-7976 (resistant parent) & $\ldots$ & & $1.4 \mathrm{a}$ & $1.4 \mathrm{a}$ & $1.0 \mathrm{a}$ \\
Trenton (susceptible parent) & $\ldots$ & & $4.2 \mathrm{~b}$ & $4.8 \mathrm{c}$ & $4.3 \mathrm{~b}, \mathrm{c}$ \\
Erik (resistant check) & $1.4 \mathrm{a}$ & & $1.4 \mathrm{a}$ & $1.4 \mathrm{a}$ & $1.4 \mathrm{a}$ \\
ND495 (susceptible check) & $3.5 \mathrm{~b}$ & & $4.2 \mathrm{~b}$ & $3.1 \mathrm{~b}$ & $3.6 \mathrm{~b}$ \\
Kulm (susceptible check) & $\ldots$ & & $4.9 \mathrm{c}$ & $4.9 \mathrm{c}$ & $4.9 \mathrm{c}$ \\
6B365 (susceptible check) & $3.6 \mathrm{~b}$ & & $5.0 \mathrm{c}$ & $4.8 \mathrm{c}$ & $4.9 \mathrm{c}$ \\
\hline
\end{tabular}

${ }^{\mathrm{z}}$ Pti2 (race 1), D308 (race 3), and 78-62 (race 1). Lesion-type means within the same column followed by the same letter are not significantly different at $P=0.05$. Lesion types range from $1=$ most resistant to $5=$ most susceptible.

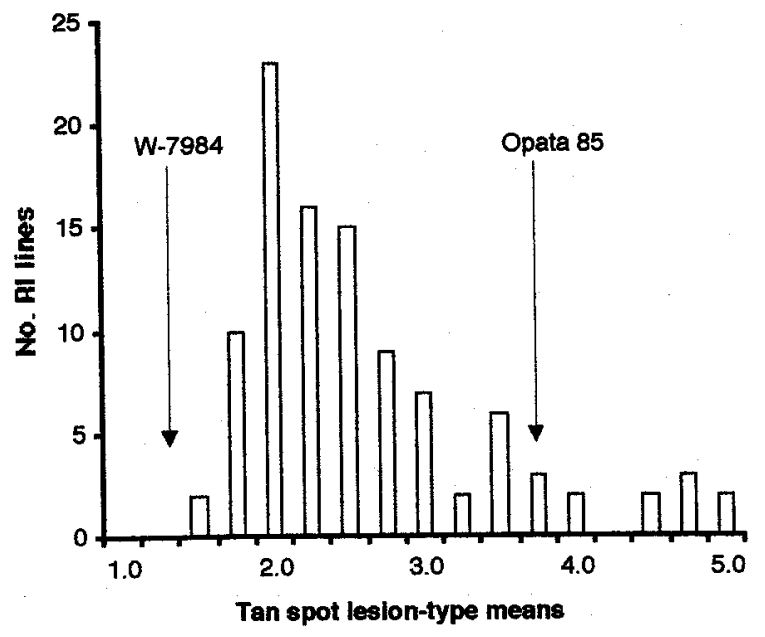

Fig. 1. Histogram of lesion-type means of adult recombinant inbred (RI) lines from the cross of W-7984/Opata 85 after inoculation of flag leaves with conidia of Pyrenophora tritici-repentis isolate Pti2. by the markers in the multiple regression model. The type I error threshold of $P=0.01$ was used for all QTL statistical analyses. The chance of declaring a nonsignificant QTL significant (type I error) was enhanced in this experiment because of the large number of RFLP-tan spot associations evaluated in the W-7984/ Opata 85 RI population. The higher probability of committing this type of error was tolerated to reduce the chance of declaring a significant QTL nonsignificant (type II error).

$\operatorname{Ptr} \operatorname{ToxA}$ purification and seedling infiltration. Approximately $10 \mu \mathrm{l}$ of Ptr ToxA $(10 \mu \mathrm{g} / \mathrm{ml})$ was infiltrated into the middle of the youngest fully expanded leaf at the third-leaf stage. Six plants each of the parents, checks, and RI lines were infiltrated with a 1-ml syringe with the needle removed. The boundaries of the infiltration area were marked with a felt pen before the water-soaked area disappeared. Infiltrated leaves were scored as sensitive or insensitive 3 days after toxin infiltration. Ptr ToxA isolation and purification was performed as described by Zhang et al. (46).

RFLP analysis of seedlings. Leaf tissue from each of the RI lines was collected for DNA extraction. DNA isolation, restriction digests, electrophoresis, Southern blotting, hybridization, and membrane washing procedures were carried out as described by Riede and Anderson (34). Forty-six low copy RFLP clones were selected on the basis of having been associated with resistance to tan spot in either the seedling (9) or adult stages.

Lesion types on the third leaf, after inoculation with each of the $P$. tritici-repentis isolates, were used to identify associations between resistance and RFLP clones. DNA from each of the RI lines was scored as one of the two parental types or as heterogeneous for segregating RFLP bands. The detection of both parental states was scored as heterogeneous. Linkage relationships were determined using the computer program MAPMAKER (version 2.0) (22) with a minimum log likelihood ratio (LOD) of 3.00 and a maximum theta equal to 0.30 .

Lesion-type means were regressed onto the RFLP marker data using the computer program DataDesk (version 4.1; Data Description Inc., Ithaca, NY) to identify loci associated with tan spot resistance at the $P<0.01$ level of probability. All of the loci with significant main effects were tested against all of the other markers to detect significant interactions $(P<0.01)$. Markers showing significant main effects and interactions were used to identify the best multiple regression model, and markers that failed to retain significance in the full model were eliminated.

\section{RESULTS}

Adult tan spot reactions. Lesion-type means of checks and parents to conidial inoculations of race $1 P$. tritici-repentis isolate Pti2 are presented in Table 1. The W-7984/Opata 85 RI population segregated for reaction to conidial inoculations with Pti2. Seedlings of this population were resistant to tan necrosis and segregated only for the extensive chlorosis symptom (9). Adults of this population also segregated for chlorosis with very little necrosis observed. Susceptible genotypes and checks exhibited chlorosis

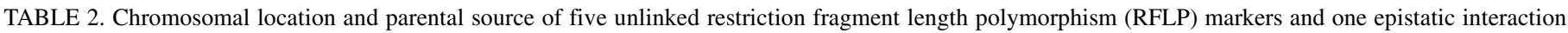

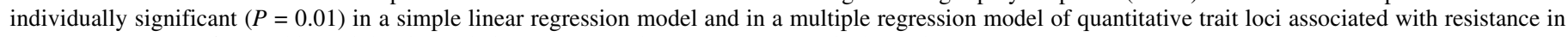
the W-7984/Opata 85 recombinant inbred population to Pyrenophora tritici-repentis isolate Pti2

\begin{tabular}{|c|c|c|c|c|c|}
\hline RFLP marker & Chromosome & Resistance source & $R^{2}$ & $P$ (single marker) & $P(\text { multiple regression model })^{\mathrm{z}}$ \\
\hline XGli1 & $1 \mathrm{AS}$ & W-7984 & 0.261 & $<0.0001$ & $<0.0001$ \\
\hline Xabc174 & $3 \mathrm{BL}$ & W-7984 & 0.090 & 0.0038 & 0.0030 \\
\hline$X b c d 1438$ & 7DS & W-7984 & 0.066 & 0.0092 & $\ldots$ \\
\hline Xksue11 & $1 \mathrm{AL}$ & Opata 85 & 0.070 & 0.0100 & 0.0003 \\
\hline Xfba351 & $5 \mathrm{AL}$ & W-7984 & 0.124 & 0.0100 & 0.0006 \\
\hline Xabc164 & $5 \mathrm{BS}$ & Opata 85 & 0.013 & 0.2606 & 0.0098 \\
\hline Xgli1 $\cdot$ Xabc1641 & $1 \mathrm{AS} / 5 \mathrm{BS}$ & $\ldots$ & 0.044 & $\ldots$ & 0.0011 \\
\hline
\end{tabular}

z Model $R^{2}=0.49$. 
surrounding the infection site as well as extensive chlorosis throughout the leaf. Resistant genotypes resembled W-7984 and cv. Erik and had small black points at the infection site with no chlorosis surrounding the infection site or elsewhere on the leaf. Variances between blocks and experiments were homogeneous, which allowed the means to be pooled. Analysis of variance indicated highly significant differences among the RI lines (data not shown).

Lesion-type means of the RI lines gave a distribution that was skewed toward resistance (Fig. 1). Most of the genotypes exhibited resistant (1 to 2) infection types or moderately resistant to moderately susceptible $(>2$ to $<4)$ with a smaller number exhibiting susceptible (4 to 5 ) infection types. This suggests that more than one gene may be involved in conditioning resistance to Pti2 in adult plants. Ten RI lines exhibited lesion types ranging from resistant to susceptible and were not included in the analysis. These lines may be heterogeneous at a major resistance locus.

Adult RFLP analysis. Lesion-type means were regressed on the RFLP marker data, resulting in the identification of five regions significantly $(P \leq 0.01)$ associated with resistance (Table 2$)$. Loci associated with resistance were contributed by both parents. The significant locus on 7DS, identified by the marker Xbcd1438, failed to remain significant in the multiple regression model. The marker Xabc164 detected a region on 5BS that was not significant by itself; however, it showed a significant epistatic interaction with XGli1. The nonsignificant locus on 5BS became significant when the interaction between the locus identified by Xabc164 and the locus identified by XGlil was put into the regression model. The main effects of XGli1, XksuE11, Xfba351, Xabc174, and $X a b c 164$, along with the interaction between XGlil and Xabc164, explained $49 \%$ of the variation for resistance to $P$. tritici-repentis isolate Pti2 in adults of the W-7984/Opata 85 RI population. The locus identified by the marker XGli1 on 1AS explained the largest portion of variation for resistance in adults at $26 \%$ (Table 2).

One significant epistatic interaction was identified in adults of this population. Progeny carrying the W-7984 allele for XGli1 exhibited the highest level of resistance regardless of which allele was carried for Xabc164 (Table 3). A high level of susceptibility was observed in genotypes that carried the Opata 85 allele at the XGli1 locus and the W-7984 allele at the Xabc164 locus. When both alleles were contributed by cv. Opata 85 , a lesser degree of susceptibility was observed. This indicated that the locus identified by Xabc164 was important in conditioning susceptibility when it was contributed from W-7984, and the XGlil locus was contributed from cv. Opata 85. However, when W-7984 contributed the allele for XGlil, the locus identified by Xabc164 was neutral. No other significant $(P=0.01)$ interactions were identified between the markers listed in Table 2 and the other markers on the map.

Seedling tan spot reactions. Lesion-type means of checks and parents for isolates Pti2, 78-62, and D308 are presented in Table 1. The W-7976/Trenton RI population segregated for both the tan necrosis and extensive chlorosis symptoms of tan spot. Susceptible genotypes and checks exhibited necrotic lesions and chloro-

TABLE 3. Lesion-type means of the four possible classifications of recombinant inbreds (RI) for allelic state at XGli1 and Xabc164 after inoculation of the population derived from the cross W-7984/Opata 85 with conidia produced by Pyrenophora tritici-repentis isolate Pti2

\begin{tabular}{llcc}
\hline XGli1 & Xabc164 & No. of RI lines & Lesion-type means ${ }^{\mathrm{z}}$ \\
\hline W-7984 & W-7984 & 24 & $2.14 \mathrm{a}$ \\
W-7984 & Opata 85 & 24 & $2.16 \mathrm{a}$ \\
Opata 85 & Opata 85 & 30 & $2.72 \mathrm{~b}$ \\
Opata 85 & W-7984 & 17 & $3.50 \mathrm{c}$ \\
\hline
\end{tabular}

${ }^{\mathrm{z}}$ Numbers followed by the same letter are not significantly different at $P=0.05$. Lesion-type means range from 1 (most resistant) to 5 (most susceptible). sis surrounding the infection site with or without extensive chlorosis throughout the leaf to isolates Pti2 and 78-62. When inoculated with isolate D308, susceptible genotypes and checks showed chlorosis surrounding the infection site with or without extensive chlorosis throughout the leaf. Resistant genotypes and checks had small black infection sites without necrosis and with no chlorosis

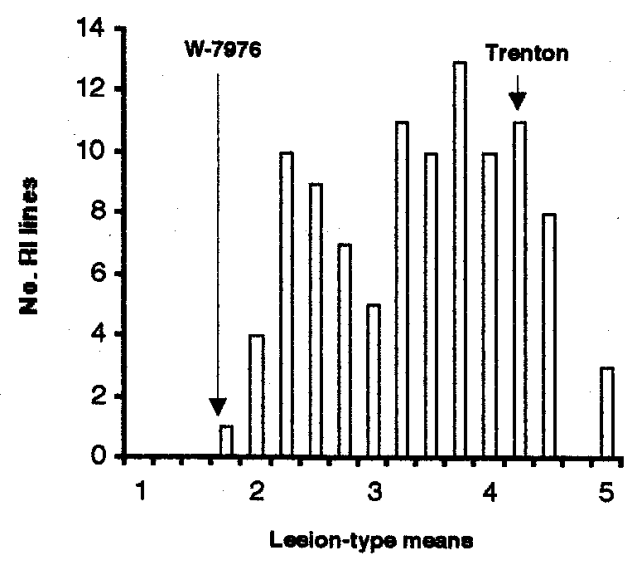

A

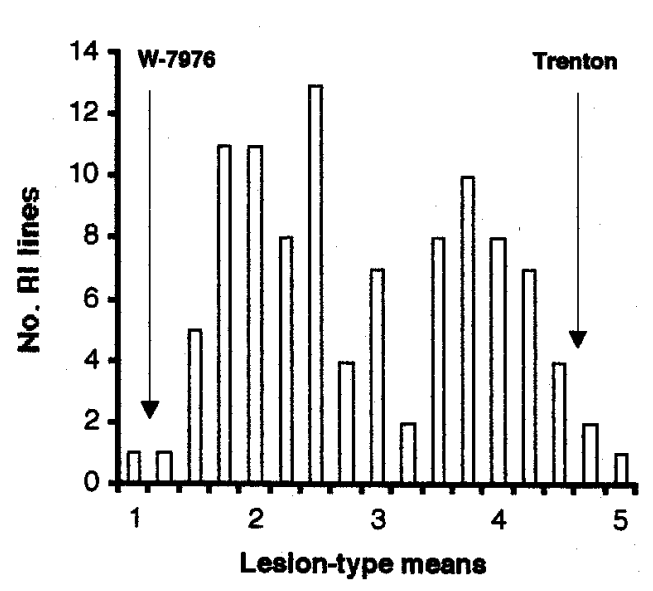

B

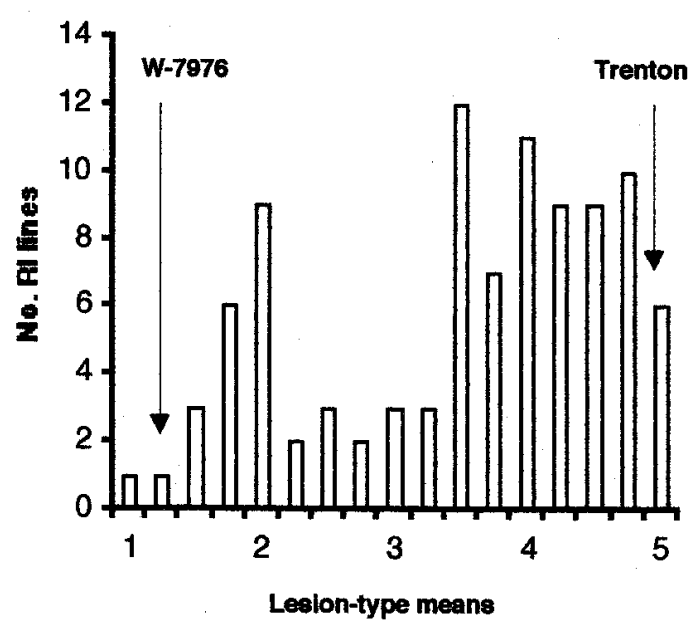

Fig. 2. Histogram of lesion-type means of seedling recombinant inbred (RI) lines from the cross of W-7976/Trenton after inoculation with conidia of Pyrenophora tritici-repentis isolates A, Pti2; B, 78-62; and C, D308. 
surrounding the infection site or elsewhere on the leaf. Variances between experiments were homogeneous, which allowed the means to be pooled. Analysis of variance indicated highly significant differences among the RI lines (data not shown).

Lesion-type means of the W-7976/Trenton RI lines for each of the three isolates resembled bimodal distributions (Fig. 2). Most of the genotypes exhibited either resistant (1 to 2) lesion types or susceptible (4 to 5) lesion types. However, several of the RI lines exhibited moderately resistant to moderately susceptible lesion types, indicating that more than one gene may be involved in conditioning resistance to these isolates in this population. A total of 10,15 , and $11 \mathrm{RI}$ lines exhibited lesion types ranging from resistant to susceptible to isolates 78-62, D308, and Pti2, respectively. These lines may be heterogeneous at a major resistance locus and were not included in the analysis.

Infiltration of purified Ptr ToxA into the RI lines produced either sensitive or insensitive reactions that closely resembled those observed in the parents and checks. No intermediate reactions were observed.

TABLE 4. Chromosomal location of polymorphic restriction fragment length polymorphism (RFLP) clones in the W-7976/Trenton recombinant inbred (RI) population

\begin{tabular}{|c|c|c|c|}
\hline RFLP clone & $\begin{array}{l}\text { Polymorphic } \\
\text { fragments }^{x}\end{array}$ & W-7984/Opata $85^{y}$ & W-7976/Trenton ${ }^{\mathrm{z}}$ \\
\hline BCD265 & 2 & $1 \mathrm{~A}, 4 \mathrm{D}$ & $\ldots$ \\
\hline CDO312 & 1 & $1 \mathrm{~A}, 1 \mathrm{~B}, 1 \mathrm{D}$ & $1 \mathrm{~A}$ \\
\hline CDO426 & 1 & $1 \mathrm{~A}$ & $1 \mathrm{~A}$ \\
\hline hor1,2 (XGli1) & 6 & $1 \mathrm{~A} .1,1 \mathrm{~A} .2,1 \mathrm{~B}, 1 \mathrm{D}$ & 1A.1, 1A.2 \\
\hline KSUD14 & 3 & 1A.1, 1A.2, 1B, 1D.1, 1D.2 & $1 \mathrm{~A}$ \\
\hline KSUE11 & 2 & 1A.1, 1A.2, 1B.1, 1B.2, 1D & $\ldots$ \\
\hline CDO346 & 2 & $1 \mathrm{~B}, 5 \mathrm{D}$ & $\ldots$ \\
\hline CDO405 & 1 & $2 \mathrm{~A}, 2 \mathrm{~B}, 2 \mathrm{D}$ & $\ldots$ \\
\hline BCD1120 & 1 & $\underline{2 \mathrm{D}}$ & $2 \mathrm{D}$ \\
\hline CDO1479 & 1 & $2 \mathrm{D}$ & $2 \mathrm{D}$ \\
\hline BCD15 & 4 & $3 \mathrm{~A}, 4 \mathrm{D}$ & $\ldots$ \\
\hline CDO460 & 1 & $3 \mathrm{~A}, 3 \mathrm{~B}$ & $\ldots$ \\
\hline BCD809 & 1 & $3 \mathrm{~B}$ & $\ldots$ \\
\hline BCD402 & 1 & $4 \mathrm{~A}, 4 \mathrm{~B}$ & $\ldots$ \\
\hline FBA78 & 3 & $4 \mathrm{~A}, 4 \mathrm{~B}$ & $4 \mathrm{~A}$ \\
\hline FBA147 & 1 & $4 \mathrm{~A}, 4 \mathrm{~B}$ & $4 \mathrm{~A}$ \\
\hline FBB1 & 1 & $4 \mathrm{~A}$ & $\ldots$ \\
\hline KSUG12 & 6 & $4 \mathrm{~A}, 7 \mathrm{~A}$ & $\ldots$ \\
\hline WG622 & 2 & $4 \mathrm{~A}, 4 \mathrm{~B}$ & $4 \mathrm{~A}$ \\
\hline BCD9 & 1 & $5 \mathrm{~B}$ & $\overline{5 \mathrm{~B}}$ \\
\hline BCD1030 & 1 & $5 \mathrm{~B}$ & $5 \mathrm{~B}$ \\
\hline FBA166 & 2 & $5 \mathrm{~A}, 5 \mathrm{~B}$ & \\
\hline FBA332 & 4 & $5 \mathrm{~B}$ & $5 \mathrm{~B}$ \\
\hline
\end{tabular}

${ }^{\mathrm{x}}$ Number of polymorphic bands resulting from hybridization of the clone with membranes of genomic DNA digested with restriction enzymes EcoRI or HindIII.

y Chromosomal location of each clone in the W-7984/Opata 85 RI population. Clones that have chromosomes underlined indicate that quantitative trait loci are associated with resistance in seedlings of that population.

${ }^{\mathrm{z}}$ The chromosomal location of unlinked clones was determined by comparing banding patterns on autoradiographs from the W-7976/Trenton RI population to autoradiographs of the W-7984/Opata 85 RI population digested with the same restriction enzyme. In cases where banding patterns were different, or different restriction enzymes were used, unlinked clones were not assigned a chromosomal location.
RFLP analysis. A total of 23 of the 46 clones surveyed detected polymorphisms and were subsequently hybridized with membranes containing digested DNA from the RI lines. All 113 RI lines were used for RFLP analysis. Forty RFLP loci were obtained from the 23 clones hybridized. Nine linkage groups were formed, and 12 markers were unlinked. The 23 clones that detected polymorphisms in the W-7976/Trenton RI population and their chromosomal locations are presented in Table 4. Fragments detected by Xfba332 were linked to the Ptr ToxA insensitivity gene, $t s n 1$, on chromosome $5 \mathrm{BL}$ at a distance of $4.4 \mathrm{cM}$ (data not shown).

Lesion-type means were regressed on the RFLP marker data to identify loci significantly associated $(P<0.01)$ with resistance to each of the $P$. tritici-repentis isolates. The marker XGlil was significantly associated with resistance to each of the isolates. The main effect seen with XGli1 had $R^{2}$ values of $0.47,0.58$, and 0.64 for isolates Pti2, 78-62, and D308, respectively. Isolate D308 was the only isolate that included more than one marker (XGlil on chromosome 1A and Xwg622 on chromosome 4A) significantly associated with resistance. A multiple regression model was developed for these markers and gave an $R^{2}$ value of 0.71 (Table 5). No significant two-locus interactions were found.

\section{DISCUSSION}

A major QTL on chromosome 1AS was associated with resistance to tan spot caused by the $P$. tritici-repentis isolate Pti2 in adult plants of the W-7984/Opata 85 RI population. The major QTL identified in adults on 1AS agreed with the location of $Q T s c . n d s u-1 A$ (9) in seedlings of this population. An interval map of this region illustrates the major effects from XGlil associated with resistance to tan spot in adults and seedlings of the W-7984/ Opata 85 RI population (Fig. 3). We conclude that this region contributes a resistance factor active throughout the life cycle of the plant.

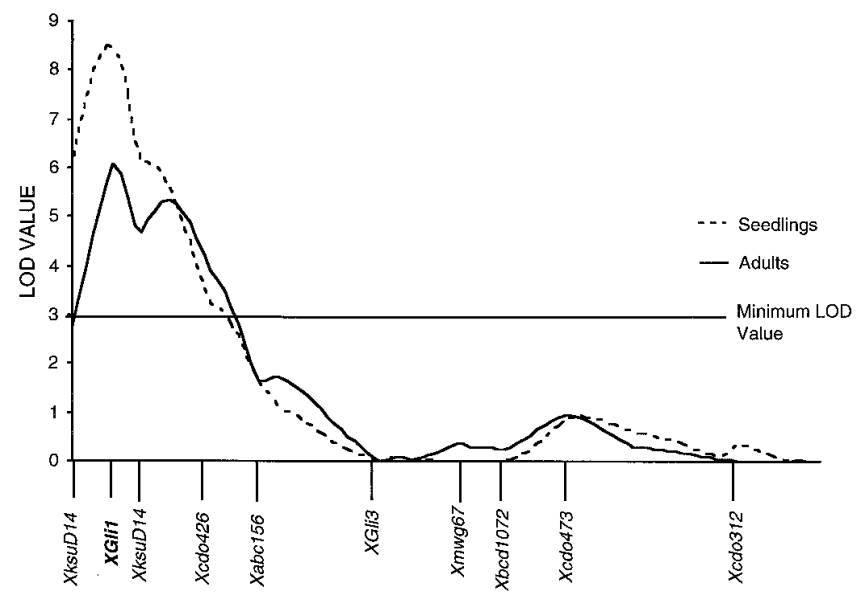

Fig. 3. Interval map of the short arm of chromosome 1A indicating the major effect from XGlil associated with resistance to tan spot caused by Pyrenophora tritici-repentis isolate Pti2 in seedlings and adults. Seedling data provided by J. Faris.

TABLE 5. Chromosomal location and parental source of unlinked restriction fragment length markers (RFLP) significant $(P=0.01)$ in a simple linear regression and in a multiple regression model of quantitative trait loci associated with resistance in the W-7976/Trenton recombinant inbred population to Pyrenophora tritici-repentis isolates Pti2, 78-62, and D308

\begin{tabular}{lcccccc}
\hline Isolate & RFLP marker & Chromosome & Resistance source & $R^{2}$ & $P$ (single marker) $^{P(\text { multiple regression) }}{ }^{\mathrm{z}}$ \\
\hline Pti2 & XGli1 & 1AS & W-7976 & 0.47 & $<0.0001$ & $\ldots$ \\
78-62 & XGli1 & 1AS & W-7976 & 0.58 & $<0.0001$ & $<$ \\
D308 & XGli1 & 1AS & W-7976 & 0.64 & $<0.0001$ & $<0.0001$ \\
D308 & Xwg622 & 4AL & W-7976 & 0.04 & $<0.0011$ & $<0.0011$ \\
\hline
\end{tabular}

${ }^{\mathrm{z}}$ Multiple regression model for isolate $\mathrm{D} 308\left(R^{2}=0.71\right)$. 
The minor QTLs identified in adults differed from those found in seedlings (9). Minor QTLs associated with resistance in adults were located on chromosomes 1AL, 7DS, 5AL, and 3BL. Chromosome $3 \mathrm{BL}$ is the only other chromosome that contained a significant QTL in both adults and seedlings; however, in the seedling population, the $3 \mathrm{BL}$ locus failed to retain significance in the multiple regression model. Faris et al. (9) identified a minor QTL on chromosome 4AL that was not found in the adult analysis. The differences found between seedlings and adults could be due to a number of reasons. Although the experiments were carried out under greenhouse conditions, the host-parasite interactions involved in tan spot are markedly affected by environment and leaf age (7). Other differences could be due to the difficulty in scoring infection types at the adult stage compared with the seedling stage. Finally, the type I error rate tolerated in these experiments can lead to spurious correlations. Evaluations of other populations are needed to confirm these minor QTLs.

A single epistatic interaction was detected in both seedlings and adults involving the major QTL detected by XGli1, however $X$ Gli1 interacted with a different locus in adults than in seedlings. The seedling interaction involved a locus on chromosome 2DL (9), and the adult interaction involved a locus on chromosome 5BS. Epistatic interactions are more difficult to identify than main effects at a single locus. This is because the linkage of markers and QTLs at more than one locus are involved, which could result in some significant epistasis going undetected. Significant QTLs in the D-genome may have gone undetected if marker-QTL linkages were not tight enough to detect significant associations due to the nine gaps of more than $20 \mathrm{cM}$ between RFLP markers.

Three isolates of $P$. tritici-repentis were screened on the $\mathrm{W}$ 7976/Trenton population, and an RFLP analysis yielded marker linkages consistent with the seedling (9) and adult populations of W-7984/Opata 85. D308 was the only isolate that was associated with a QTL marker other than XGli1, and no epistatic interactions among markers within the population were seen with any of the three isolates. The low number of clones hybridized to the RI lines in this experiment could account for missing other QTLs associated with resistance to tan spot. We were able, however, to confirm the association of QTsc.ndsu-1A to resistance to several isolates of $P$. tritici-repentis in a different genetic background than previously reported.

The W-7976/Trenton population segregates for both tan necrosis and extensive chlorosis symptoms of tan spot. RFLP markers from regions that were reported to be associated with these symptoms and were polymorphic in this population were included in this study. This included all of the markers found to be significantly associated with resistance by Faris et al. $(8,9)$ and those found to be associated with resistance in adult plants of the W7984/Opata 85 RI population. Unfortunately, not all of the markers found to be associated with resistance in the W-7984/ Opata 85 RI population were polymorphic in the W-7976/Trenton RI population. In these cases, flanking markers were used until a polymorphic marker was identified. This may be the reason for the identification of only one minor QTL associated with resistance to tan spot in this population. However, this QTL, detected by Xwg622 on chromosome 4AL, may be the same as that detected by Faris et al. (9). A larger mapping effort would be necessary in the W-7976/Trenton RI population to identify other minor QTLs significantly associated with resistance to tan spot. To identify novel QTLs, it may be useful to develop a population derived from a resistance source other than the two synthetic hexaploids used (W-7984 and W-7976).

Selection for resistance only to extensive chlorosis will not lead to complete resistance for tan spot. Tan necrosis is also a virulence component of this disease and should be selected for as well. For both Pti2 and 78-62 but not D308, the W-7976/Trenton population segregated for necrosis and was screened with Ptr ToxA. The clone $X f b a 322$ segregated in this population and was linked to the toxin insensitivity gene, tsnl, on chromosome 5BL. However, only the locus associated with resistance to extensive chlorosis was significantly associated with resistance to conidial inoculations with the two race 1 isolates used in this study. This may indicate that extensive chlorosis is the more destructive symptom. Selection for resistance to tan necrosis has been a much easier process due to the availability of Ptr ToxA, which has been associated with the tan necrosis symptom, and the fact that resistance to tan necrosis is conditioned by a single gene. Infiltration of agronomically acceptable genotypes with Ptr ToxA and determining sensitivity to the toxin is an easy screening method for this symptom.

Screening for extensive chlorosis is much more difficult because extensive chlorosis is quantitatively inherited. The development of polymerase chain reaction-derived molecular markers linked to QTLs associated with resistance to extensive chlorosis could facilitate a marker-assisted selection scheme that would enable breeders to screen lines for resistance. Results of this study confirm that resistance to conidia inoculations of $P$. tritici-repentis isolates are associated with QTsc.ndsu-1A. This locus has been associated with resistance to tan spot in three studies and has accounted for 35 (9) to $64 \%$ of the variation for resistance in two populations of seedlings and $26 \%$ of the variation for resistance in adults. This would indicate that incorporating the QTsc.ndsu-1A locus, along with the $t s n 1$ locus, would result in wheat lines more resistant to tan spot than the predominant cultivars grown in the Great Plains region of the United States and Canada.

\section{ACKNOWLEDGMENTS}

This research was partially supported by the U.S. Department of Agriculture-National Research Institute grant 93-37311-9388 awarded to J. A. Anderson and L. J. Francl.

\section{LITERATURE CITED}

1. Ballance, G. M., Lamari, L., and Bernier, C. C. 1989. Purification and characterization of a host selective toxin from Pyrenophora triticirepentis. Physiol. Mol. Plant Pathol. 35:203-213.

2. Ballance, G. M., Lamari, L., Kowatsch, R., and Bernier, C. C. 1996. Cloning, expression and occurrence of the gene encoding the Ptr necrosis toxin from Pyrenophora tritici-repentis. Mol. Plant Pathol. (published by the British Society for Plant Pathology). On-line Publication no. 1996/1209ballance.

3. Brown, C. A., and Hunger, R. M. 1993. Production of a chlorosisinducing, host-specific, low-molecular weight toxin by isolates of Pyrenophora tritici-repentis, cause of tan spot of wheat. J. Phytopathol. 137:221-232.

4. Ciuffetti, L. M., Tuori, R. P., and Gaventa, J. M. 1997. A single gene encodes a selective toxin causal to the development of tan spot on wheat. Plant Cell 9:135-144.

5. Cox, D. J., and Hosford, R. M., Jr. 1987. Resistant winter wheats compared at differing growth stages and leaf positions for tan spot severity. Plant Dis. 71:883-886.

6. da Luz, W. C., and Hosford, R. M., Jr. 1980. Twelve Pyrenophora trichostoma races for virulence to wheat in the Central Plains of North America. Phytopathology 70:1193-1196.

7. Elias, E., Cantrell, R. G., and Hosford, R. M., Jr. 1989. Heritability of resistance to tan spot in durum wheat and its association with other agronomic traits. Crop Sci. 29:299-304.

8. Faris, J. D., Anderson, J. A., Francl, L. J., and Jordahl, J. G. 1996. Chromosomal location of a gene conditioning insensitivity in wheat to a necrosis-inducing culture filtrate from Pyrenophora tritici-repentis. Phytopathology 86:459-463.

9. Faris, J. D., Anderson, J. A., Francl, L. J., and Jordahl, J. G. 1997. RFLP mapping of resistance to chlorosis induction by Pyrenophora triticirepentis in wheat. Theor. Appl. Genet. 94:98-103.

10. Fernandez, M. R., Clarke, J. M., and DePauw, R. M. 1994. Response of durum wheat kernels and leaves at different growth stages to Pyrenophora tritici-repentis. Plant Dis. 78:597-600.

11. Haley, C. S., and Knott, S. A. 1992. A simple regression method for mapping quantitative trait loci in line crosses using flanking markers. Heredity 69:315-324.

12. Hallock, Y. F., Lu, H. S. M., Clardy, J., Strobel, G. A., Sugawara, F., 
Samsoedin, R., and Yoshida, S. 1993. Triticones, spirocyclic lactams from the fungal plant pathogen Drechslera tritici-repentis. J. Nat. Prod. 56:747-754.

13. Hosford, R. M., Jr. 1982. Tan spot-developing knowledge 1902-1981. Pages 1-5 in: Tan Spot of Wheat and Related Diseases Workshop. R. M. Hosford Jr., ed. North Dakota State University, Fargo.

14. Hosford, R. M., Jr., Jordahl, J. G., and Hammond, J. J. 1990. Effect of wheat genotype, leaf position, growth stage, fungal isolate, and wet period on tan spot lesions. Plant Dis. 74:385-390.

15. Krupinsky, J. M. 1982. Observations of the host range of isolates of Pyrenophora trichostoma. Can. J. Plant Pathol. 4:42-46.

16. Lamari, L., and Bernier, C. C. 1989. Evaluation of wheat lines and cultivars to tan spot [P. tritici-repentis] based on lesion type. Can. J. Plant Pathol. 11:49-56.

17. Lamari, L., and Bernier, C. C. 1989. Toxin of Pyrenophora triticirepentis: Host-specificity, significance in disease, and inheritance of host reaction. Phytopathology 79:740-744.

18. Lamari, L., and Bernier, C. C. 1991. Genetics of tan necrosis and extensive chlorosis in tan spot of wheat caused by Pyrenophora triticirepentis. Phytopathology 81:1092-1095.

19. Lamari, L., Bernier, C. C., and Ballance, G. M. 1992. The necrosischlorosis model in tan spot of wheat. Pages 10-15 in: Advances in Tan Spot Research. L. J. Francl, J. M. Kuprinsky, and M. P. McMullen, eds. North Dakota State Univ. Agric. Exp. Stn., Fargo.

20. Lamari, L., Bernier, C. C., and Smith, R. B. 1991. Wheat genotypes that develop both tan necrosis and extensive chlorosis in response to isolates of Pyrenophora tritici-repentis. Plant Dis. 75:121-122.

21. Lamari, L., Sayoud, D., Boulif, M., and Bernier, C. C. 1996. Identification of a new race in Pyrenophora tritici-repentis: Implications for the current pathotype classification system. Can. J. Plant Pathol. 17:312-318.

22. Lander, E. S., Green, P., Abrahamson, J., Barlow, A., Daly, M. J., Lincoln, S. E., and Newburg, L. 1987. MAPMAKER: An interactive computer package for constructing primary genetic linkage maps of experimental and natural populations. Genomics 1:174-181.

23. Lee, T. S., and Gough, F. J. 1984. Inheritance of Septoria leaf blotch ( $S$. tritici) and Pyrenophora tan spot ( $P$. tritici-repentis) resistance in Triticum aestivum cv. Carifen 12. Plant Dis. 68:848-851.

24. Marino, C. L., Nelson, J. C., Lu, Y. H., Sorrells, M. E., Leroy, P., Tuleen, N. A., Lopes, C. R., and Hart, G. E. 1996. Molecular genetic maps of the group-6 chromosomes of hexaploid wheat (Triticum aestivum L. em. Thell.). Genome 39:359-366.

25. Nagle, B. J., Frohberg, R. G., and Hosford, R. M., Jr. 1982. Inheritance of resistance to tan spot of wheat. Pages 40-45 in: Tan Spot of Wheat and Related Diseases Workshop. R. M. Hosford Jr., ed. North Dakota State University, Fargo.

26. Nelson, J. C. 1997. QGENE: Software for marker-based genomic analysis and breeding. Mol. Breed. 3:239-245.

27. Nelson, J. C., Sorrells, M. E., Van Deynze, A. E., Lu, Y. H., Atkinson, M., Bernard, M., Leroy, P., Faris, J. D., and Anderson, J. A. 1995. Molecular mapping of wheat. Major genes and rearrangements in homoeologous groups 4, 5, and 7. Genetics 141:721-731.

28. Nelson, J. C., Van Deynze, A. E., Autrique, E., Sorrells, M. E., Lu, Y. H., Merlino, M., Atkinson, M., and Leroy, P. 1995. Molecular mapping of wheat. Homoeologous group 2. Genome 38:516-524.
29. Nelson, J. C., Van Deynze, A. E., Autrique, E., Sorrells, M. E., Lu, Y. H., Negre, S., Bernard, M., and Leroy, P. 1995. Molecular mapping of wheat. Homoeologous group 3. Genome 38:525-533.

30. Orolaza, N. P., Lamari, L., and Ballance, G. M. 1995. Evidence of a host-specific chlorosis toxin from Pyrenophora tritici-repentis, the causal agent of tan spot of wheat. Phytopathology 85:1282-1287.

31. Rees, R. G., and Platz, G. J. 1983. Effects of yellow spot on wheat: Comparison of epidemics at different stages of crop development. Aust. J. Agric. Res. 34:39-46.

32. Rees, R. G., and Platz, G. J. 1990. Sources of resistance to Pyrenophora tritici-repentis in bread wheats. Euphytica 45:59-69.

33. Rees, R. G., and Platz, G. J. 1992. Tan spot and its control-some Australian experiences. Pages 1-9 in: Advances in Tan Spot Research. L. J. Francl, J. M. Krupinsky, and M. P. McMullen, eds. North Dakota State University, Fargo.

34. Riede, C. R., and Anderson, J. A. 1996. Linkage of RFLP markers to an aluminum tolerance gene in wheat. Crop Sci. 36:905-909.

35. Riede, C. R., Francl, L. J., Anderson, J. A., Jordahl, J. G., and Meinhardt, S. W. 1996. Additional sources of resistance to tan spot of wheat. Crop Sci. 36:771-777.

36. Shoemaker, R. A. 1962. Drechslera Ito. Can. J. Bot. 40:810-836.

37. Steffenson, B. J., Hayes, P. M., and Kleinhofs, A. 1996. Genetics of seedling and adult plant resistance to net blotch (Pyrenophora teres $\mathrm{f}$. teres) and spot blotch (Cochliobolus sativus) in barley. Theor. Appl. Genet. 92:552-558.

38. Stock, W. S., Brûlé-Babel, A. L., and Penner, G. A. 1996. A gene for resistance to a necrosis-inducing isolate of Pyrenophora tritici-repentis located on 5BL of Triticum aestivum cv. Chinese Spring. Genome 39: 598-604.

39. Strelkov, S. E., Lamari, L., and Balance, G. M. 1999. Characterization of a host-specific protein toxin (Ptr ToxB) from Pyrenophora tritici-repentis. Mol. Plant-Microbe Interact. 12:728-732.

40. Sykes, E. E., and Bernier, C. C. 1991. Qualitative inheritance of tan spot resistance in hexaploid, tetraploid, and diploid wheat. Can. J. Plant Pathol. 13:38-44.

41. Tomás, A., and Bockus, W. W. 1987. Cultivar specific toxicity of culture filtrate of Pyrenophora tritici-repentis. Phytopathology 77:1337-1340.

42. Tuori, R. P., Wolpert, T. J., and Ciuffetti, L. M. 1995. Purification and immunological characterization of toxic components from cultures of Pyrenophora tritici-repentis. Mol. Plant-Microbe Interact. 8:41-48.

43. Van Deynze, A. E., Dubcovsky, J., Gill, K. S., Nelson, J. C., Sorrells, M. E., Dvorak, J., Gill, B. S., Lagudah, E. S., McCouch, S. R., and Appels, R. 1995. Molecular-genetic maps for group 1 chromosomes of Triticea species and their relation to chromosomes in rice and oat. Genome 38: 45-59.

44. Watkins, J. E., Odvody, G. N., Boosalis, M. G., and Partridge, J. E. 1978. An epidemic of tan spot of wheat in Nebraska. Plant Dis. Rep. 62: 1232-1234.

45. Zhang, H. 1997. A Pyrenophora tritici-repentis necrosis toxin: Protein isolation and characterization and gene cloning and expression. Ph.D. diss. North Dakota State University, Fargo.

46. Zhang, H.-F., Francl, L. J., Jordahl, J. G., and Meinhardt, S. W. 1997. Structural and physical properties of a necrosis-inducing toxin from Pyrenophora tritici-repentis. Phytopathology 87:154-160. 